\title{
The Preventing Harassment in Science Workshop: Summary and Best Practices for Planetary Science and Astrobiology
}

Authors: Kristen Bennett [1], Maggie McAdam [2], Moses Milazzo [3], Patricia Garcia [1], Jenna Shelton [1], Peggy Gardiner [1], Serina Diniega [4], Catalina Martinez [6], Alex Etheridge [1], Alicia Rutledge [7], Christina Richey [4]. (corresponding author email: kbennett@usgs.gov)

Co-signers: Frances Rivera-Hernández [8], Michaela Leung [9], Timothy A. Goudge [10], Michael A. Mischna [4,5], Jennifer Hanley [11], Cynthia Phillips [4,5], Ryan Watkins [12], Steven A. Hauck, II [13], Rebecca J. Smith [14], Antonella Alunni [2], Noel A. Scudder [15], Kerri Donaldson Hanna [16], Caela Barry [17, 18], Jordyn-Marie Dudley[19, 20], Akos Kereszturi [21, 22], Timothy D. Glotch [14], Ariel N. Deutsch [23], Marcella Yant [24], Krista Soderlund [10], Angela G Marusiak [4, 5], Nicole Whelley [25, 18], Jennifer Scully [5,4], Jacob Richardson [25], Alessondra Springmann [26], Nancy Chabot [27], Romy D. Hanna [10], Antonio J. Ricco [2, 28], Linda Spilker [4], Jamie L. Molaro [12], Marisa Mayer [28], Alfred S. McEwen [26], Kunio M. Sayanagi [29], Kelsi N. Singer [30], Evan L. Sneed [31], Jeffrey M. Moore [2], Noam R. Izenberg [27], Colin Dundas [1], Gwen Hanley [32]; Gabrielle Engelmann-Suissa [33].

Affiliations: [1] U.S. Geological Survey; [2] NASA Ames Research Center; [3] OtherOrb; [4] Jet Propulsion Laboratory; [5] California Institute of Technology; [6] NOAA; [7] Northern Arizona University; [8] Dartmouth College; [9] University of California, Riverside; [10] The University of Texas at Austin;

[11] Lowell Observatory; [12] Planetary Science Institute; [13] Case Western Reserve University; [14] Stony Brook University; [15] Purdue University; [16] University of Central Florida; [17] ADNET Systems, Inc.; [18] NASA GSFC; [19] Jacobs; [20] JETS, at NASA Johnson Space Center; [21] CSFK; [22] Konkoly Observatory; [23] Brown University; [24] Lockheed Martin; [25] University of Maryland College Park; [26] University of Arizona; [27] JHU Applied Physics Laboratory; [28] Stanford University; [29] Hampton University; [30] Southwest Research Institute; [31] Pennsylvania State University; [32] University of California Berkeley; [33] University of Washington.

This white paper is submitted as part of a collaborative effort organized by the Equity, Diversity, and Inclusion Working Group (EDIWG), a cross Assessment Group (AG) committee

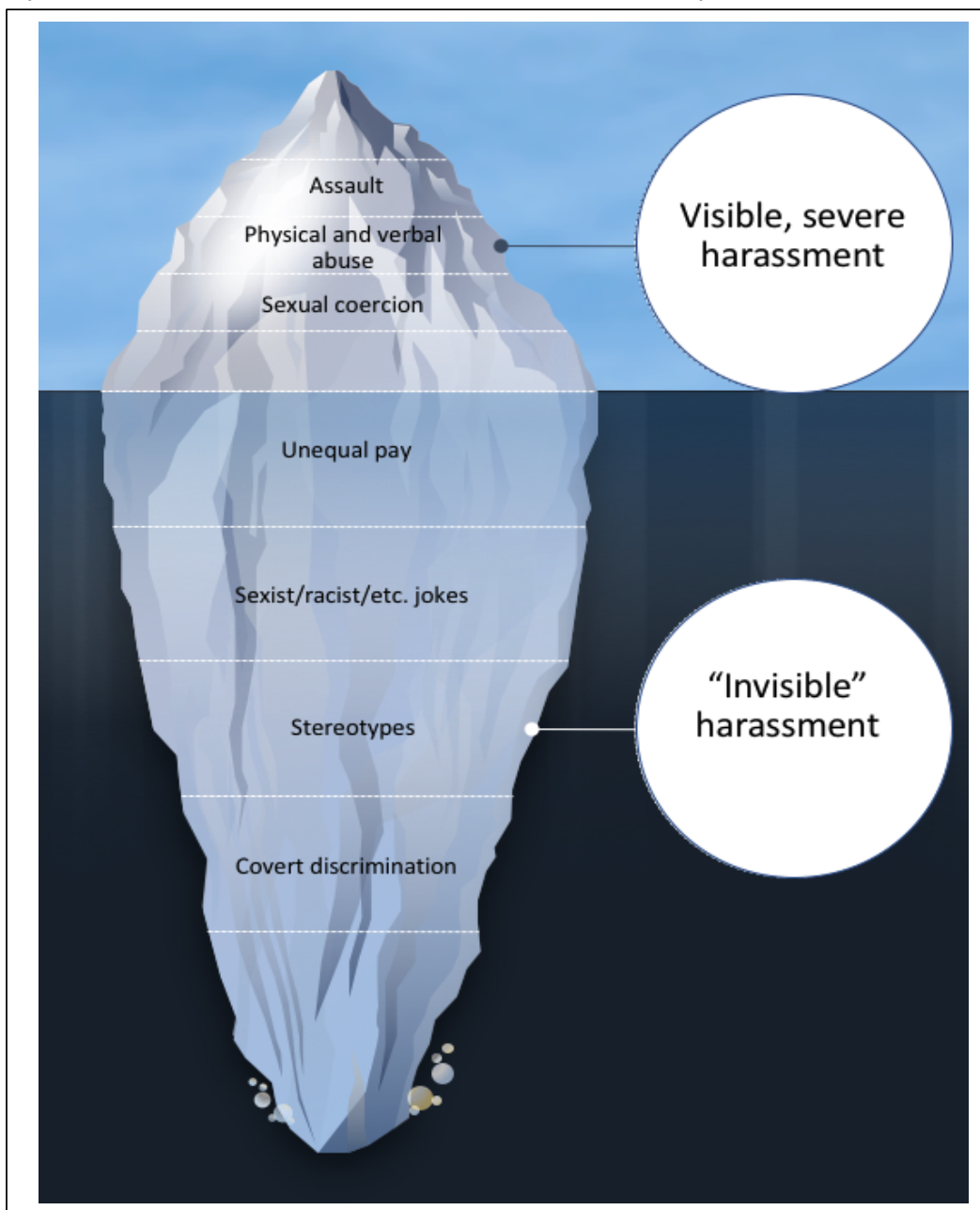

Cover Figure: Types of harassment visualized as an Iceberg. Iceberg from: https://templates.office.com/en-us/iceberg-graphic-tm01003742 


\section{Introduction}

The Preventing Harassment in Science: Building a Community of Practice for Meaningful Change workshop took place virtually in June 2020. This highly successful, NASA-funded workshop brought leaders of anti-harassment efforts together to share ideas and discuss best practice methods to reduce harassment in the scientific workplace.

Throughout the workshop, we explicitly acknowledged the historic moment through which we are collectively living. The global COVID-19 pandemic loomed large for this event. In the days and weeks before the workshop, the protests sparked by the killings of George Floyd, Breonna Taylor and Ahmaud Arbery were ongoing and fresh in our minds. Dr. Aradhna Tripati of UCLA described this moment as 'the Third Reconstruction.' Like previous moments in history, after the Civil War and during the Civil Rights Era, we as a society are in tension between the struggle to bend the moral arc of history towards justice and the resistance to change in favor of the status quo. This workshop was in and of itself another site of this struggle. Implicit in the conception of this event was the injustice of the systems and structures in Science, Technology, Engineering, and Math (STEM) that have created and continue to support the slow rolling crisis of harassment in our field. Many people in STEM are struggling against these systems and structures. This workshop was a chance for us to gather and collectively 'reimagine our spaces' as Dr. Tripati put it and work toward justice in STEM.

In this white paper, we describe the workshop and summarize the best practices for reducing harassment that were discussed. We include a list of actions that can be used to reduce harassment and increase inclusion in the planetary science and astrobiology community. Additionally, a list of resources from the workshop can be found here ${ }^{1}$.

\section{Summary of the Workshop}

\subsection{Origins}

Vision Statement: This workshop will bring leaders of anti-harassment efforts together to share ideas and discuss best practice methods to reduce harassment in the workplace.

The Preventing Harassment in Science (PHIS) workshop originated as an effort to centralize anti-harassment efforts and share best practices, primarily focusing on government and academic spaces. Many people in science are individually combating harassment and creating inclusive spaces. One goal of this workshop was to facilitate conversations between people who have developed strategies to reduce harassment, including social scientists. Participants could share their work and experiences, and best anti-harassment practices would be compiled for widespread use. This white paper addresses the goal of compiling a list of actions that can be used to reduce harassment (see section 4).

This workshop focused on harassment but necessarily also addressed inclusion. A hostile or non-inclusive environment leads to harassment. For this reason, we determined it was critical to include discussion of inclusivity practices in an anti-harassment workshop.

The organizing committee ${ }^{2}$ consisted of employees from U.S. Geological Survey (USGS), NASA, and NOAA. Funding for this event was provided by NASA through the NASA-USGS PSDI Interagency Agreement (NNH17AE04I). We are immensely grateful to NASA for

${ }^{1}$ https://docs.google.com/document/d/1qZX7zg9OdZQvszlExjkeOCp1oFj15wW2bQCcepApyK4/edit?usp= sharing

${ }^{2}$ https://www.hou.usra.edu/meetings/anti-harassment2020/organizers/ 
financially supporting anti-harassment efforts. Because NASA funded the event and many of the organizers are planetary scientists, the workshop included an emphasis on the field of planetary science; therefore, the output of this workshop is highly relevant to this Decadal Survey.

\subsection{Response to COVID-19}

The PHIS workshop was initially planned as an in-person meeting in late March 2020. When stay-at-home orders were implemented across the USA in mid-March due to COVID-19, the in-person workshop was cancelled. Upon switching to a virtual meeting to take place June 2425 , the focus of the workshop necessarily changed. The original expectation of the workshop was to bring together leaders in anti-harassment to network, share ideas and experiences, and compile a list of anti-harassment best practices. We had hoped that some science community members would join to learn about anti-harassment efforts in person, but recognized that many scientists do not have the funding to travel to workshops such as this one. With the pivot from a small $(<50$ person) intimate workshop to a virtual meeting, we were able to reach a much broader audience ( 400 people). While the virtual event reduced the amount of networking and small group discussions, we believe these negative aspects were outweighed by the positive impact of reaching a broader audience.

We worked with Lunar and Planetary Institute (LPI) meeting planners to organize the workshop. We are grateful to LPI for their meeting support services. When we asked if LPI would be willing to support a large, virtual meeting, they responded enthusiastically and worked tirelessly to facilitate the virtual experience.

Despite the quarantine and communal efforts to stem the spread of the pandemic, violence against Black people did not stop. Perhaps unsurprisingly, with communities already on edge and suffering due to COVID-19, new examples of egregious violence against Black people were met with outrage and protests of historic numbers. One additional consequence of postponing our workshop was that it allowed us to respond to the Black Lives Matter protests directly and reflect on them as a part of this event, albeit unintentional timing of the two events. The summer of 2020 is proving to be a turning point in regards to Americans prioritizing and acknowledging systemic racism and inclusion in their workplaces and their lives. We heard from many registrants that they were motivated to attend the PHIS workshop because of the protests and the newly elevated discussion around systemic racism. The PHIS workshop embraced this challenging discussion, and we believe that workshops such as this can be utilized to address major issues and work towards positive change.

\subsection{Brief summary of the workshop}

The PHIS workshop took place on June 24 and 25, 2020. There were over 400 total registrants with more than 150 people dialed into the workshop at any given time. An online discussion platform was used to engage participants, share ideas, network, and solicit questions for the speakers. The full schedule and additional information are available on the workshop website. ${ }^{3}$

The first day focused on actions that people and institutions have taken and can take to reduce harassment. This included a talk on building effective codes of conduct, a presentation from the National Academies of Sciences, Engineering, and Medicine Action Collaborative on Preventing Sexual Harassment in Higher Education (NASEM, 2018), and examples of how to build a harassment prevention program at an institution from the ground up. A panel discussed

${ }^{3}$ https://www.hou.usra.edu/meetings/anti-harassment2020/program/ 
how institutions respond to harassment, both negatively ("Passing the Harasser") and positively (i.e., enacting positive change related to anti-harassment policies). A second panel focused on grassroots anti-harassment initiatives started by individuals.

The second day focused on anti-harassment training and inclusion. The first panel brought together experts in anti-harassment training to discuss effective training techniques. The second panel focused on the planetary science community and how to increase inclusivity in this specific field. Next, the documentary "Can We Talk?" by Kendall Moore was aired, followed by a Q\&A session. This documentary focuses on the lack of belonging felt by many people of color in STEM fields and how this pushes many people out of the field. Finally, Dr. Kathryn Clancy of the University of Illinois, Champaign-Urbana, facilitated a workshop on how to increase inclusivity in your community.

\section{Key Takeaways and Best Practices for Reducing Harassment 3.1 Key Takeaway: "Legal compliance is necessary but not sufficient." -Dr. Alex Helman, NASEM}

The concept that legal compliance is necessary but insufficient to combat harassment was first brought up by Dr. Helman and echoed throughout the workshop. Demonstrating that harassment will not be tolerated through codes of conduct and policy is incredibly important for institutional leadership. Without open support for anti-harassment efforts from institutional leaders, the culture and climate of the community likely will not improve. That said, having antiharassment policies is not enough to reduce harassment.

One of the speakers, Dr. Clancy, spoke about the difference between a workplace culture and climate. Workplace culture includes the written rules of the workplace or organization, for example, the mission statement or vision statement of the organization as well as required training, policies and expected behaviors. Workplace climate, on the other hand, is the lived experience that employees have and how they feel about the organization. Incentives and rewards, condoned and condemned behavior and the unwritten rules are captured in workplace climate. The difference between culture and climate reflects the idea that legal compliance is necessary but not sufficient. If a workplace has appropriate policies and culture statements in place, it is still possible for a negative or toxic climate to exist. Both culture and climate need to be addressed in order to improve the workplace environment.

\subsection{Key Takeaway: "If you don't make a big deal out of the small things, when the big things come your voice will be too small." -Erica White-Dunston, Chief Diversity Officer of Department of Interior}

Following on the concept that legal compliance isn't enough is the idea that inappropriate behavior must be stopped before it reaches the severity of illegal harassment. Harassment has many definitions depending on the target and the nature of the harassment, but here we will use a paraphrased definition ${ }^{5}$ from the USGS: illegal harassment is " unwelcome conduct ... that is sufficiently severe or pervasive as to create a work environment that a reasonable person would consider intimidating, hostile, or abusive."

Illegal harassment is the tip of the inappropriate iceberg (i.e. cover page figure). Allowing small inappropriate behaviors, such as racist or sexist jokes, to become normalized then allows the

\footnotetext{
${ }^{4}$ https://www.kendallmooredocfilms.com/can-we-talk

${ }^{5}$ USGS anti harassment policy: https://www.usgs.gov/about/organization/sciencesupport/survey-manual/3707341-anti-harassment-policy-and-implementing accessed 9/1/2020
} 
inappropriate behavior to escalate to become more severe forms of harassment. This is where techniques such as Bystander Intervention can play an important role in creating a positive work environment. In this theory, individuals must take on the responsibility to act when they witness inappropriate behavior, no matter how small it seems. This demonstrates that inappropriate behavior will not be tolerated in the workplace and results in fewer occurrences of the inappropriate behaviors.

\subsection{Key Takeaway: "Bad apples vs. rotten barrel framing" - Dr. Clancy.}

Dr. Clancy also redefined our current framework for understanding harassment, especially sexual harassment, and challenged us to consider these incidents differently. We habitually see high-profile and commonplace incidents of sexual harassment in a 'bad apples' framework. This framework focuses on the individual who targets people with their power. The individual person is 'bad' and their removal is the solution to the problem of harassment in the workplace/organization. Rather than this outlook, the 'rotten barrel' framing looks at the people around a harasser as well as the systems and structures in place that enable their bad behavior. Harassers exist in communities of complex systems that are not set up to support targets of harassment. This may include a lack of a clear channel to report harassment or reporting/investigating processes that have backlogs or otherwise take a very long time. Furthermore, these systems are often punitive (rather than restorative) and the harassment must be sufficiently severe to warrant investigation. Harassers also exist in communities where peers do not challenge minor transgressive behaviors thus allowing harassers to escalate their transgressions with impunity. Finally, community members see and choose to ignore small incidents of bad behavior for a variety of reasons. These reasons include a reluctance to start a conflict with their peer, fear of retaliation from the harasser if the harasser has seniority, or a desire for a collegial environment. These fears and desires are perhaps understandable but when we choose our personal comfort over transgressions by individual community members, we choose to allow harassers to hurt people around us, especially those with less power or status. When we start thinking of the rotten barrel, we are moved to use our voices differently and to restructure our workplaces to ensure everyone can work in freedom and safety from harassment.

\subsection{Key Takeaway: Top down, bottom up, and disruptive changes}

When thinking about how to change a community's culture and who should be responsible to change the culture, there are three important aspects.

Top down: It is critical for the leaders of a community to be actively involved in positive change. This is related to section 3.1, where we learned that if effective policies are not in place, none of these efforts will work. Management needs to champion efforts to reduce harassment. The community needs to see that management is supportive (both financially and of new ideas to change workplace culture and/or climate). From the grassroots panel on Day 2, we heard from 
employees who said that their grassroots inclusion efforts would not have succeeded without people in leadership positions championing their cause.

Bottom up: Many inclusion and anti-harassment efforts start with one or two motivated individuals in a community that may or may not serve in a leadership role. The culture cannot change without the active involvement of community members who are working for change. Examples of "bottom up" grassroots efforts include peercreated and peer-led Bystander Intervention workshops, Peer Support Workers who help targets of harassment to navigate the reporting chain, and support groups for people of various identities.

Innovation, creativity, and disruption: Communities have been fighting harassment and working on diversity and inclusion

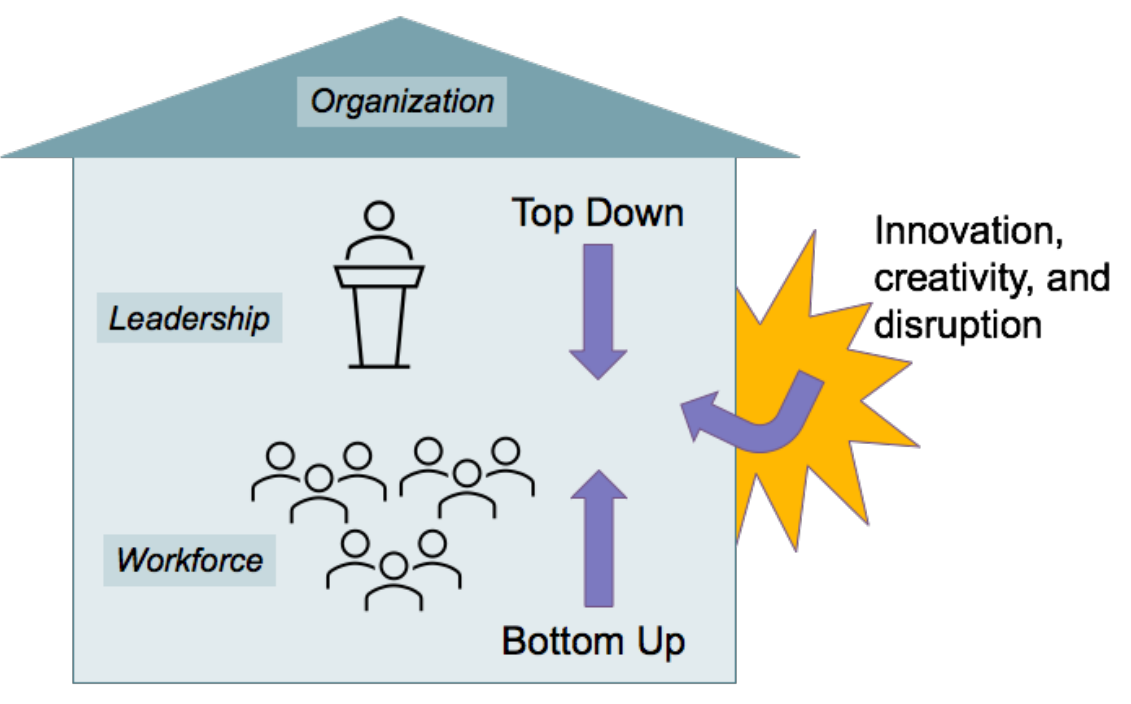
issues for decades in STEM. There is no magic solution. Nothing that has been tried has been fully successful. This means that we need to be open to new, potentially disruptive, ideas. We encourage everyone to avoid resisting an idea just because it has never been done before. An example from the workshop was the idea from Sheila O'Rourke of UC Davis to include background checks and disclosure of personnel files from previous institution(s) in faculty hiring in an attempt to stop the "Pass the Harasser" problem; "Pass the Harasser" refers to when a faculty member gets fired for harassing behaviors, and then immediately rehired somewhere else, potentially allowing and/or enabling their bad behavior to continue. Another example of this is to get a perspective outside of your immediate community to learn how to implement new, innovative strategies. For example, NOAA hired Kelly Bonner, an expert in anti-harassment efforts, to rebuild their harassment prevention program from the ground up.

\subsection{Key Takeaway: "Compassion does not mean comfort" - Kelly Bonner, NOAA}

The necessary disruptive changes to address harassment and inappropriate behavior in STEM need to be made with compassion for everyone. We are moved to address the systemic and structural oppression in STEM out of compassion for our peers who unjustly struggle to be in STEM fields. These changes must also be made with compassion to those who do not experience the negative effects of systemic harassment in STEM. For people who are surprised upon learning of the crisis of harassment in STEM, the call to act against harassment can feel overwhelming, perhaps even unfair. Dr. Kelly Bonner of NOAA remarked that "compassion is not the same as comfort." We must extend empathy and compassion to everyone; however, this does not mean that we have the right to comfort. Indeed, the appeal to comfort often is an appeal to embrace the status quo - to resist change. Finding a way to accept discomfort without deflecting, derailing, or altogether halting a conversation is imperative to face and address the crisis of harassment in STEM. 


\section{Actions that can reduce harassment in the Planetary Science and Astrobiology community.}

Based on the remarks of the speakers, panelists and organizers, as well as the robust discussion by the participants, we conclude that implementing the following actions can reduce harassment in STEM organizations and workplaces:

Action Item 1: Bravery, boldness, innovation. Organizations are challenged to be brave and bold and develop innovative ways to address systemic and structural factors that allow harassment to occur in STEM. Harassment is entrenched in our fields and it is imperative that new approaches are used. This requires organizations to take risks and along with this the need for bravery and boldness.

Action Item 2: Trainings: Trainings are an essential tool for supporting positive and inclusive workplace cultures and climates. By requiring these trainings, we indicate that our workplaces value and prioritize equity, inclusion and justice. The trainings that were suggested include Trauma Informed Responder Training for managers and mandatory reporters, Bystander Intervention Training, and Micro-aggressions workshop. Programs focusing on managers and supervisors are necessary as well as awareness programs for all employees.

Action Item 3: Codes of conduct. Similarly, codes of conduct are another method for creating an inclusive and just workplace culture and climate, particularly when they are designed with intentional, appropriate ramifications for violations thereof. Codes of conduct define the values of the institution/workplace and give everyone a touchstone to recognize and help respond to both unintentionally harmful and malicious behaviors. Codes of conduct allow organizations to connect with their values in an intentional way. For more information, see Diniega et al. (2020).

Action Item 4: Work with social scientists. While we know there is a crisis of harassment within STEM, we do not fully understand the impacts or the experiences of those impacted. In order to understand and amend the crisis of harassment in STEM, speakers at our workshop suggested working with social scientists. Skilled professionals in the social sciences have the necessary tools and experience (that physical scientists often lack) to capture the nature and extent of harassment and related structures that support a culture of harassment. Social scientists can provide insights on how to address harassment in STEM. Crucially, the work of social scientists investigating STEM will enable us to better pursue the scientific enterprise.

Action Item 5: Culture and climate assessments. Comprehensive organizational culture and climate assessments with associated implementation plans and measurable rubrics to determine effectiveness and improvement can specifically address the ongoing crisis of harassment. This work builds on Action Items 1 - 4 and could greatly improve organizations in STEM with respect to solving the crisis of harassment.

Action Item 6: Value service work. Service work consists of non-scientific duties that allow science to happen. Duties include reviewing papers, serving on committees, mentoring, education and public outreach, and doing the meaningful work described in this list above (i.e., giving and taking trainings, creating a code of conduct, working with social scientists). This work is usually unpaid but much of it is an expectation of paid scientific employment. One key observation from the workshop, backed by research (e.g., Buch et al., 2011; Miller et al., 2020), is that women, 
especially Black, Latina, and Indigenous women in STEM are asked to and often do significantly more service work than their peers, and then they are punished for this during evaluation and promotion. One bold idea is to allow scientists to do service work as a part of their funding proposals and include this in success/promotion criteria. Furthermore, normalize or encourage proposers to budget time and money for trainings (see above). For more information, see Rathbun et al. (2020).

Action Item 7: Continue holding and funding anti-harassment workshops in the future. The work of this vibrant community of resistance is ongoing. Brave, bold, innovative changes are being attempted all the time in our field and new guidance and "best practices" come from social science. Holding workshops such as this one annually or bi-annually can allow more focused discussion, including smaller topical workshops intended to address specific aspects of harassment and/or inclusion.

Acknowledgements: The authors of this white paper thank NASA for funding this workshop, LPI for providing technical support, and the USGS for additional institutional support. Additionally, we thank Kendall Moore and NOAA for screening the "Can We Talk?" documentary. A portion

of the work described was carried out at the Jet Propulsion Laboratory, California Institute of Technology, under a contract with the National Aeronautics and Space Administration (80NM0018D0004).

\section{References:}

Buch, K., Huet, Y., Rorrer, A. and Roberson, L., 2011. Removing the barriers to full professor: A mentoring program for associate professors. Change: The Magazine of Higher Learning, 43(6), pp.38-45.

Diniega, S. et al., 2020 Ensuring a safe and equitable workplace: the importance and feasibility of Codes of Conduct. White Paper submitted to the Planetary Science Decadal Survey.

Miller, C. and Roksa, J., 2020. Balancing research and service in academia: Gender, race, and laboratory tasks. Gender \& Society, 34(1), pp.131-152.

National Academies of Sciences, Engineering, and Medicine (NASESM). 2018. Sexual Harassment of Women: Climate, Culture, and Consequences in Academic Sciences, Engineering, and Medicine. Washington, DC: The National Academies Press. doi: https://doi.org/10.17226/24994.

Rathbun et al., 2020. Enabling the Planetary Workforce to do the best science by funding work that is a service to the Profession White Paper submitted to the Planetary Science Decadal Survey. 